\title{
SYNCHROTRON RADIATION INDUCED X-RAY EMISSION - SRIXE
}

\author{
W.M. KWIATEK \\ Institute of Nuclear Physics, Department of Nuclear Spectroscopy \\ Radzikowskiego 152, 31-342 Kraków, Poland
}

The synchrotron radiation induced X-ray emission (SRIXE) technique was found very useful and sensitive for determination of trace elements content and distribution in different types of materials. Due to properties of synchrotron radiation the SRIXE technique became very unique and powerful for trace elements analysis. This paper describes the phenomena related to production of characteristic X-rays and principles of the method. The properties of SRIXE such as minimum detectable limit, spatial resolution, radiation damage, and depth sensitivity are also discussed. Selected applications are given to emphasize the usefulness of the technique.

PACS numbers: 78.70.En, 87.15.Mi

\section{Introduction}

The elemental composition of different materials can be determined by means of many techniques, but none of them is so powerful as synchrotron radiation induced X-ray emission (SRIXE). Due to many advantages of synchrotron radiation the SRIXE technique became very popular.

The physics of such a method is quite similar to the XRF technique except for the primary photon beam intensity and possible use of "white" beam.

\section{Interaction of photon beam}

A photon beam can interact with a target through the photoelectric effect, through Compton or Rayleigh scattering. The attenuation of the beam is relatively weak so that for energies used in SRIXE experiments the beam can produce electron shell vacancies in a relatively large depth of target material. This is illustrated in Fig. 1, which shows the attenuation of a $15 \mathrm{keV}$ photon beam as a function of the distance from the surface in polyethylene, which can be taken to approximate a biological material.

Since the atomic inner-shell vacancy was created, the atom emits characteristic X-rays, which are measured in order to identify and calculate the elemental composition of the analyzed material. 


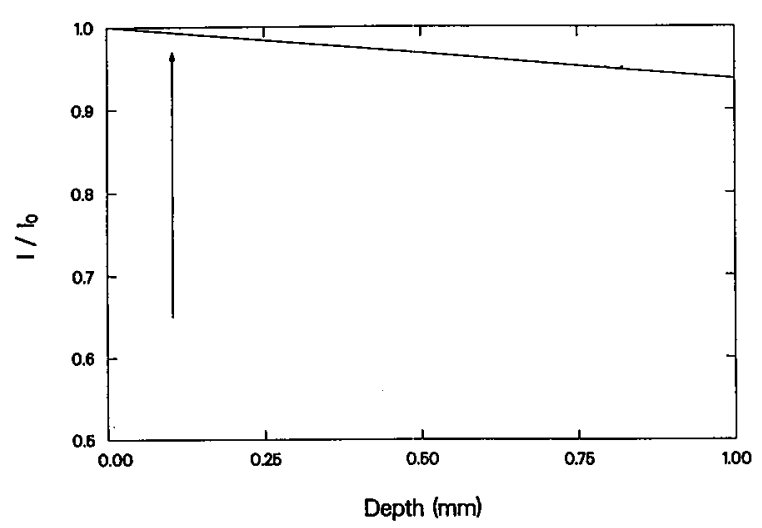

Fig. 1. Attenuation of $15 \mathrm{keV}$ photon beam as a function of the distance from the surface in polyethylene in comparison to $2.5 \mathrm{MeV}$ proton beam range marked by an arrow.

\section{Production of focussed photon beam}

A key factor in the use of SRIXE technique is the flux of incident photons which reach the target. This will determine the sensitivity of the measurement. The flux produced can be improved by adding focussing elements in the beam transport system.

As an example of what is possible with a synchrotron X-ray source, let us consider the X-26C beam line at the National Synchrotron Light Source (NSLS). The sample is located at $20 \mathrm{~m}$ from the $\mathrm{X}$-ray source. The photon flux, integrated over all energies above $3.5 \mathrm{keV}$ in the white beam, is about $4 \times 10^{7}$ photons $/\left(\mathbf{s} \cdot \mu \mathrm{m}^{2}\right)$. In order to assess properly the X-ray production rates for this case, the energy-dependent product of photon flux and vacancy production cross-section should be evaluated. Results of such a calculation are shown in Fig. 2 as a function of target atomic number.

It is also possible to use a monoenergetic photon beam. If this is done and the energy of the incident beam is $15 \mathrm{keV}$, the value of $1.8 \times 10^{6}$ photons $/\left(\mathrm{s} \cdot \mu \mathrm{m}^{2}\right)$ is found for the photon flux at the target. In this case the energy of the beam can be adjusted to optimize the photon production by choosing a value close to the absorption edge.

The important point to outline is that synchrotron radiation is a very brilliant source of X-rays. In this case we mean that brilliance is the number of photons emitted into unit solid angle per unit source area per second. Values for the DORIS storage ring in DESY in Hamburg are shown in Fig. 3.

Several different focussing systems were proposed and used on keV-energy $\mathrm{X}$-ray beams. The Daresbury microprobe used Bragg diffraction with a focussing graphite crystal, Oak Ridge employs a two-stage system, and IBM/MIT and others use $1: 1$ grazing incidence focussing mirror and a multilayer monochromator. The BNL X-26 beam line group worked on the production of an $8: 1$ focussing mirror [1] in an attempt to push the grazing incidence technique to its limits. Underwood 


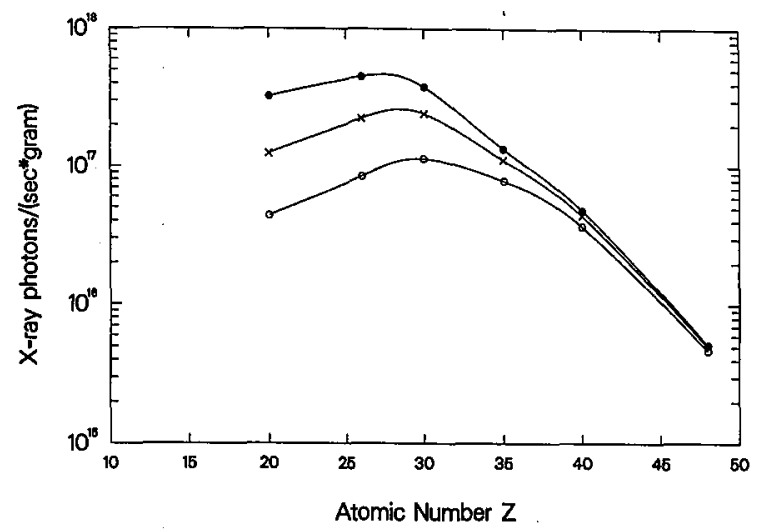

Fig. 2. Energy-dependent product of photon flux and vacancy production cross-section as a function of target atomic number. The presented guide lines correspond to different Al filters: dots $-100 \mu \mathrm{m}$, crosses $-200 \mu \mathrm{m}$, rings $-400 \mu \mathrm{m}$.

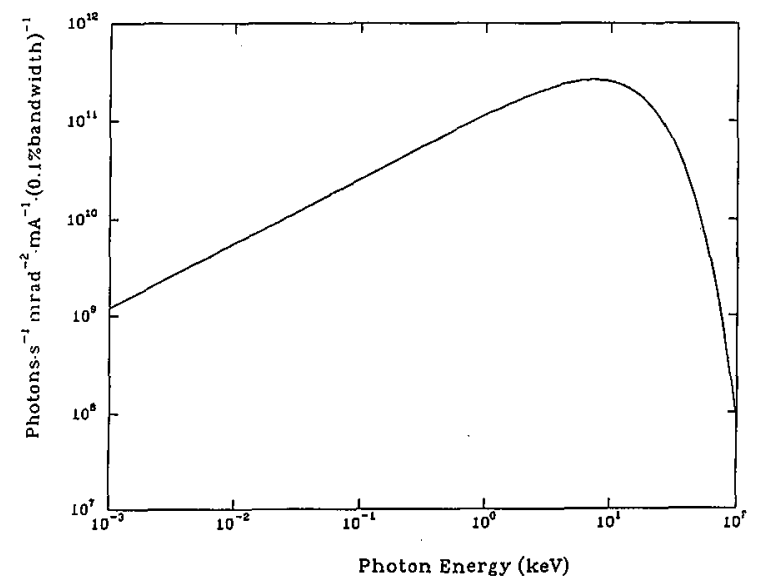

Fig. 3. Brilliance for DORIS storage ring $\left(3.7 \mathrm{GeV}, \psi=0^{\circ}\right)$.

et al. [2] made a two-stage monochromator-focussing device using multilayer mirrors which can be used to diminish the image of the primary beam produced by a preliminary focussing device which serves to transport the X-ray object to some useful working distance from the $\mathrm{X}$-ray ring. The most recent focussing system has been applied at DORIS storage ring in Hamburg [3] by Swedish group from Göteborg. Such a system is based on a capillary and the total reflection principle [4].

It can be seen that the use of synchrotron radiation makes it possible to provide either white or monoenergetic X-rays at a sample with photon fluxes that are in the neighbourhood of $10^{11}$ photons $/\left(\mathrm{s} \cdot \mathrm{cm}^{2}\right)$. 


\section{Production of characteristic X-rays}

The production of characteristic X-rays depends on the cross-section for vacancy production and the fluorescence yield giving the probability of $\mathrm{X}$-ray emission when the vacancy is filled. The relative magnitudes of the product of cross-section $\left(\sigma_{K}\right.$ or $\left.\sigma_{L}\right)$ and fluorescence yield $\left(w_{K}\right)$ should then be compared for the two processes as one crucial factor in determining the relative detection sensitivities. The needed information on $K$-or $L$-absorption cross-sections is tabulated by Krause et al. [5] and for $w_{K}$ by Bambynek [6].

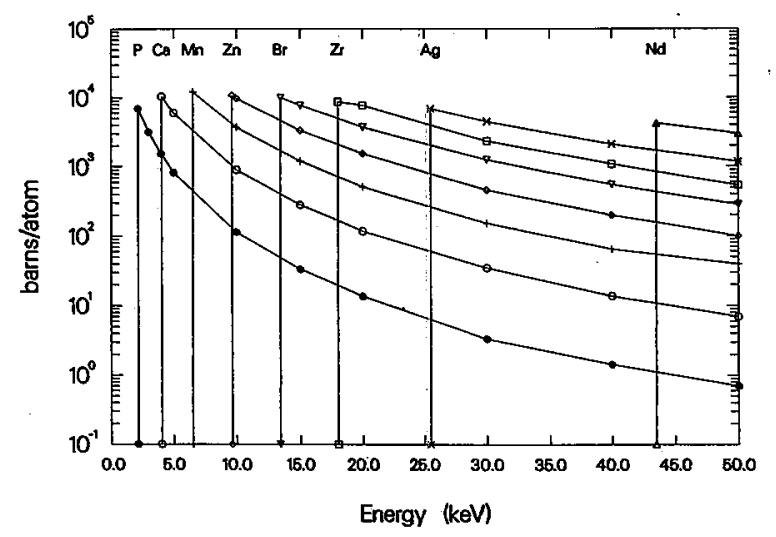

Fig. 4. Product of fluorescence yield and cross-section as a function of photon energy for selected elements.

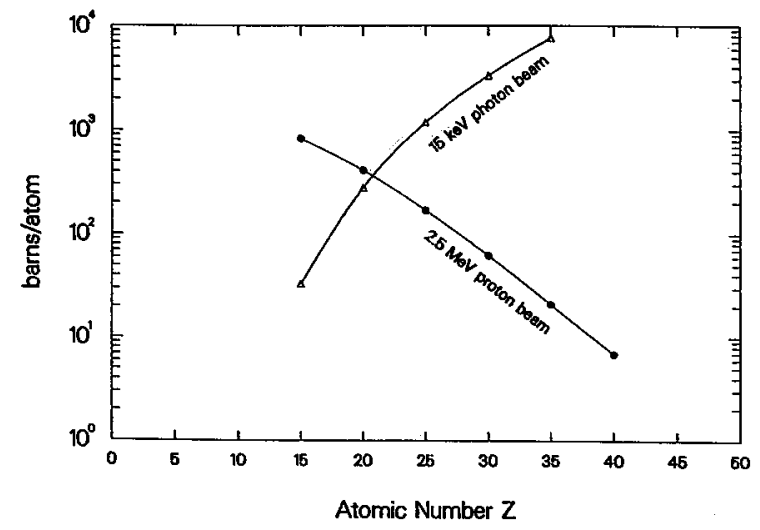

Fig. 5. The production cross-section as a function of the target atomic number at a fixed photon energy in comparison to $2.5 \mathrm{MeV}$ proton beam production cross-section. 
The product of fluorescence yield and cross-section is shown in Fig. 4 as a function of photon energy for selected elements. The production cross-sections are also shown as a function of the target atomic number at fixed photon energy in Fig. 5.

\section{Background}

In SRIXE spectra the background can be produced mainly by scattered incident photon beam. As an example, Fig. 6 shows a spectrum of a thin biological

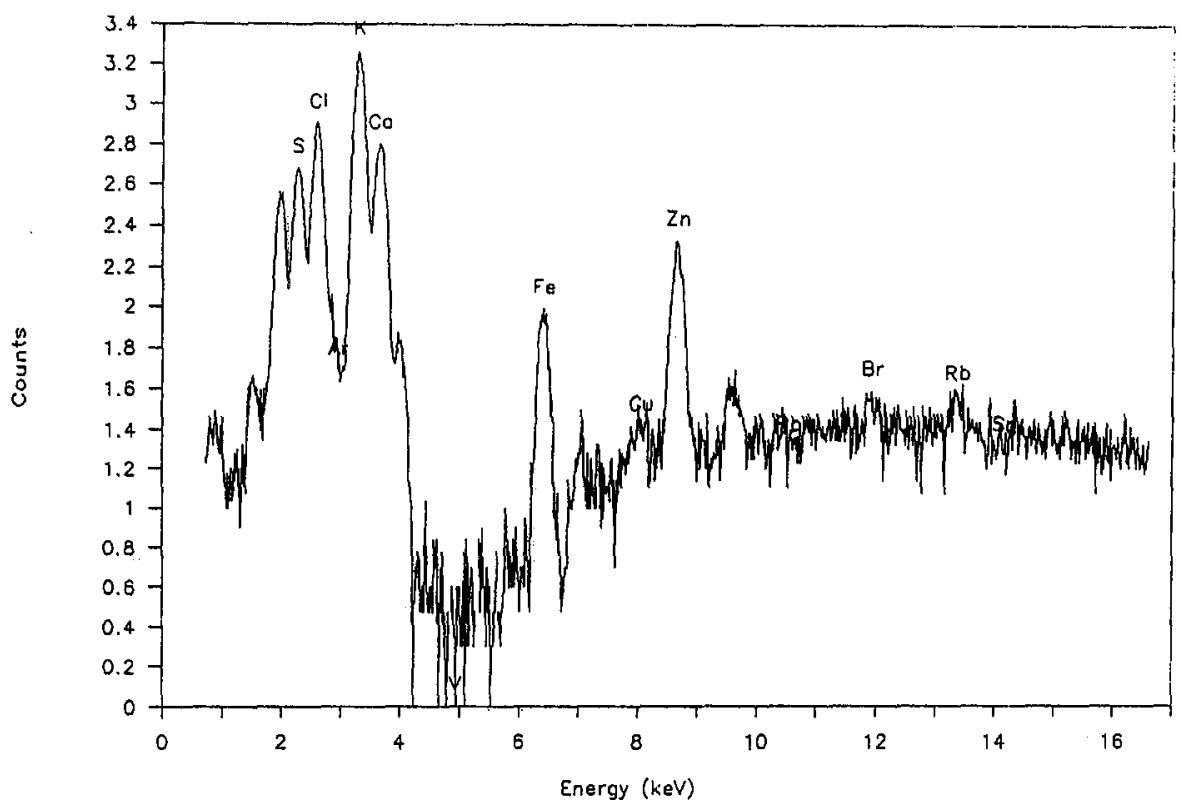

Fig. 6. SRIXE spectrum of a thin biological sample - kidney section.

sample which was a section of a kidney. The spectrum was taken with white photon beam. The SRIXE spectrum shows clearly the effect of the continuous scattered radiation from the white beam.

The production rates for the background were estimated by several authors: Sparks [7], Gordon [8], Grodzins [9], and the others.

\section{Minimum detectable limit}

The minimum detectable limit (MDL) is usually cited as a figure which characterizes the detection system. MDL is usually defined by following Currie criteria [10] as

$$
\mathrm{MDL}=3.29 C \sqrt{B} / N
$$

where $C$ - concentration of the element $Z, B$ - background area under the peak for the element $Z, N$ - net area under the peak for the element $Z$. 
Care must be taken in making comparisons of MDL's, since several different quantities enter into them. One could include experimental conditions as an example: beam size, spectrum acquisition time, incident photon flux (ring current), and sample size. Therefore, the comparisons of MDL's can be provided only for normalized spectra to the same experimental conditions. As an example of MDL curve, Fig. 7 shows such a calculated curve for the biological sample measured at the NSLS white beam.

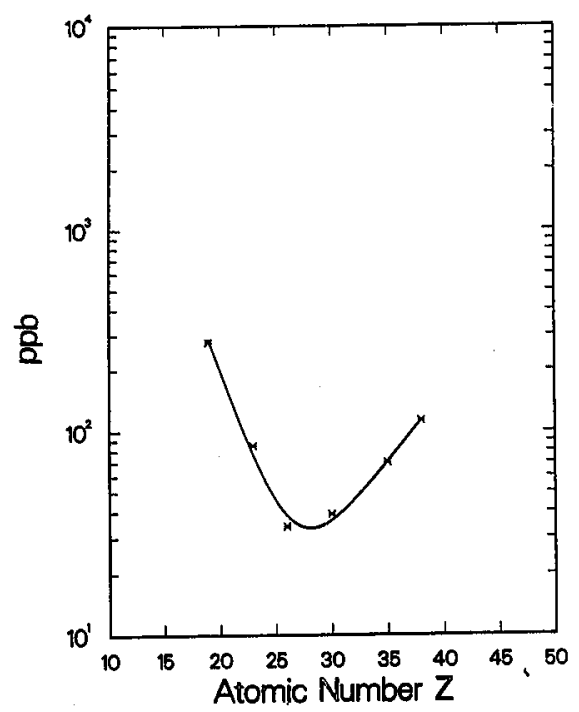

Fig. 7. MDL-fitted theoretical curve for biological sample measured at NSLS while beam. The curve is described by a function of atomic number $Z: F(Z)=$ $\exp \left(a_{1}-a_{2} Z\right)+\exp \left(-a_{3}+a_{4} Z\right)$, where in this case $a_{1}=12.56, a_{2}=0.36, a_{3}=$ $1.38, a_{4}=0.16$. The data points are marked by crosses.

\section{Spatial resolution}

An important aspect of the SRIXE technique is the ability to make trace elements measurements with excellent spatial resolution. The values which were achieved with traditionally focussed X-rays are around $1-5 \mu \mathrm{m}$ in diameter but using capillary system are even down to $0.1 \mu \mathrm{m}$, while with collimated beam the values are around $10-20 \mu \mathrm{m}$.

\section{Radiation damage}

The use of any type of microprobe is limited by the radiation damage caused in the sample by the probing beam. Approximate estimates of the relative radiation damage caused by proton beams and photon beams can be done using well-known 
values for stopping powers for ions and for absorption coefficients for X-rays. Such an estimate was done by Sparks [7] who found that the damage caused by photon beams was as much as two orders of magnitude less than that caused by proton beams. The effect of radiation damage to the targets was a subject of many papers. Such an effect was studied by Cholewa et al. [11] and it shows the importance of its consideration.

\section{Depth sensitivity}

It is not always feasible to use thin targets. For example, geological samples are difficult to prepare in thicknesses of a few micrometers. Interpretatiun of data from thicker targets may be difficult because of heterogeneity of the sample if there is no depth sensitivity. In material studies it may be of interest to measure the distribution of trace elements as a function of depth below surface.

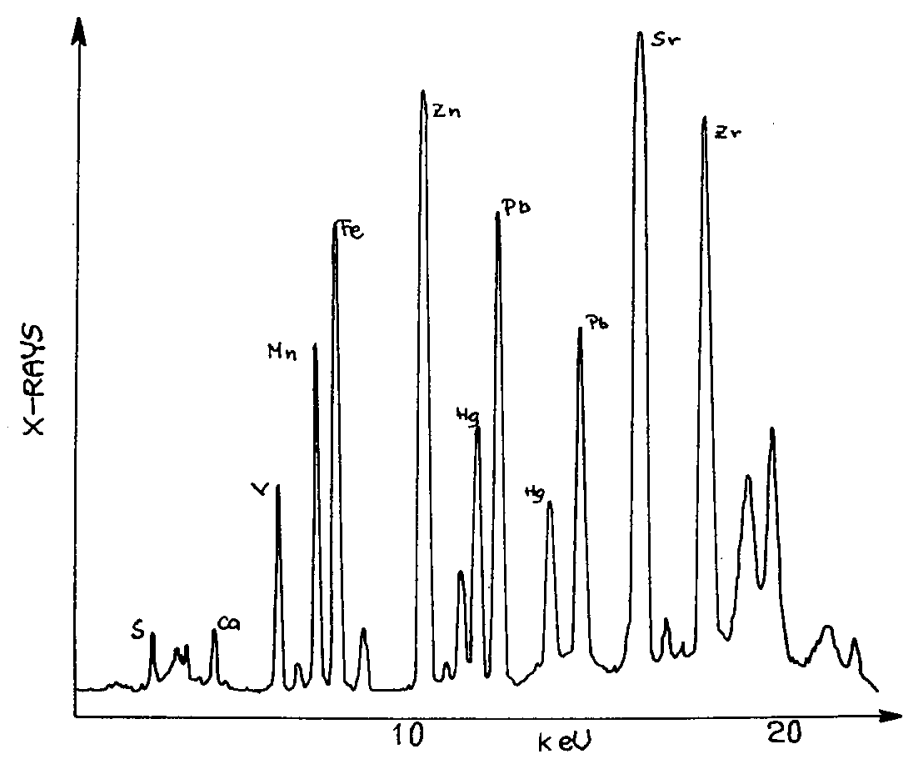

Fig. 8. TRXRF spectrum of a thin biological sample measured at Hamburg University.

Photons are weakly attenuated and are not suitable for doing depth-dependent measurements in a "classical" geometry. They can be applied, however, for doing measurements of the surface concentrations using their reflection from a surface. For a small grazing angle of incidence, the beam is totally reflected and interacts only with the surface atoms of the material. This is obviously very dependent on the condition of the surface and needs a very smooth finish to be effective [12].

The principle [13] was applied at DORIS storage ring by Rindby's group. The background of scattered photons is almost eliminated, while the fluorescent $\mathrm{X}$-rays are not affected. The MDL's are dropped down to $\mathrm{ppb}$ range. 
A typical TRXRF spectrum of a thin biological section is shown in Fig. 8. This is a very elegant technique which merits wide application.

\section{Determination of concentration}

Methods of determination of the concentration are of primary importance. Most experiments which were done with PIXE or SRIXE used standard reference materials to establish concentration scales. In such a case much care has to be taken during the measurements due to experimental conditions which have to be normalized for both standard reference material and sample measurements.

The final X-rays spectrum is described by a formula

$$
Y=N_{\mathrm{t}}(\Omega / 4 \pi) \varepsilon w \exp \left(-\mu_{\mathrm{a}}(E) x_{\mathrm{a}}\right) \int_{0}^{\infty} \int_{0}^{X} N_{\mathrm{p}}(E) \sigma(E) \exp (-\mu x) \mathrm{d} x \mathrm{~d} E,
$$

where $Y$ - total yield for an element, $N_{\mathrm{t}}$ - number of atoms in a target within a beam volume, $\Omega / 4 \pi$ - detector solid angle, $\varepsilon$ - detector efficiency, $w$ - fluorescence yield, $\mu_{\mathrm{a}}$ - linear attenuation coefficient for detector filter, $x_{\mathrm{a}}$ - detector filter thickness, $X$ - target thickness, $N_{\mathrm{p}}$ - number of irradiating photons, $E-$ energy of irradiating photons, $\sigma-$ ionization cross-section, $\mu-$ mass attenuation coefficient for a target matrix.

The above formula does not include the polarization factor. One has to consider the polarization effect since it has a great influence to the background of a spectrum.

The importance of an alignment procedure was described in several papers [14].

\section{Summary}

The aspects considered were: properties of photon beam, production of photon beams, production of characteristic X-rays, backgrounds, minimum detection limits, spatial resolution, radiation damage, depth sensitivity, and determination of concentration. It can be concluded that SRIXE is an extremely powerful technique for trace elements analysis which has many substantial positive features.

The work presented in this paper was partially performed and supported by both Brookhaven National Laboratory, Upton, New York, USA and HASYLAB at DESY in Hamburg, Germany.

\section{References}

[1] K.W. Jones, P.Z. Takacs, J.B. Hastings, J.M. Casstevens, C.D. Pionke, in: Proc. SPIE Meeting, Los Angeles 1987.

[2] H.J. Underwood, A.C. Thompson, Y. Wu, T.W. Barbee Jr., Nucl. Instrum. Methods Phys. Res. $B$ (1986).

[3] A. Buttkewitz, F. Grabe, G. Gaul, A. Knochel, F. Lechtenberg, P. Engstron, S. Larson, A. Rindby, in: HASYLAB Progress Report, HASYLAB, Hamburg 1990, p. 577.

[4] R. Klockenkamper, Spectrom. Int. 2, 26 (1990). 
[5] M.O. Krause, C.W. Nestor Jr., C.J. Sparks Jr., E. Ricci, in: Oak Ridge National Laboratory Report-5399, ORNL, Oak Ridge 1978.

[6] W. Bambynek, Rev. Mod. Phys. 44, 716 (1972).

[7] C.J. Sparks Jr., in: Synchrotron Radiation Research, Eds. H. Winick, S. Doniach, Plenum Press, New York 1980, p. 459.

[8] B.M. Gordon, Nucl. Instrum. Methods 204, 223 (1982).

[9] L. Grodzins, NeuroTox. 4, 23 (1983).

[10] L.A. Currie, Anal. Chem. 40, 586 (1968).

[11] M. Cholewa, G. Bench, B.J. Kirby, G.J.E. Legge, Nucl. Instrum. Methods Phys. Res. B 54, 101 (1991).

[12] G.H. Vineyard, Phys. Rev. B 26, 4146 (1982).

[13] A. Rindby, P. Engstrom, S. Larson, B. Stocklassa, X-ray Spectrometry 18, 109 (1989).

[14] W.M. Kwiatek, A.L. Hanson, K.W. Jones, Nucl. Instrum. Methods Phys. Res. B 50, 347 (1990). 